\title{
Early-time spectra of supernovae and their precursor winds
} \section{The luminous blue variable/yellow hypergiant progenitor of SN 2013cu}

\author{
Jose H. Groh
}

\author{
Geneva Observatory, Geneva University, Chemin des Maillettes 51, CH-1290 Sauverny, Switzerland \\ e-mail: jose.groh@unige.ch
}

Received 22 August 2014 / Accepted 10 November 2014

\begin{abstract}
We present the first quantitative spectroscopic modeling of an early-time supernova ( $\mathrm{SN}$ ) that interacts with its progenitor wind. Using the radiative transfer code CMFGEN, we investigate the recently reported $15.5 \mathrm{~h}$ post-explosion spectrum of the type IIb SN 2013cu. We are able to directly measure the chemical abundances of a SN progenitor and find a relatively H-rich wind, with $\mathrm{H}$ and He abundances (by mass) of $X=0.46 \pm 0.2$ and $Y=0.52 \pm 0.2$, respectively. The wind is enhanced in $\mathrm{N}$ and depleted in $\mathrm{C}$ relative to solar values (mass fractions of $8.2 \times 10^{-3}$ and $1.0 \times 10^{-5}$, respectively). We obtain that a slow, dense wind or circumstellar medium surrounds the precursor at the pre-SN stage, with a wind terminal velocity $v_{\text {wind }} \lesssim 100 \mathrm{~km} \mathrm{~s}^{-1}$ and mass-loss rate of $\dot{M} \simeq$ $3 \times 10^{-3}\left(v_{\text {wind }} / 100 \mathrm{~km} \mathrm{~s}^{-1}\right) M_{\odot} \mathrm{yr}^{-1}$. These values are lower than previous analytical estimates, although $\dot{M} / v_{\infty}$ is consistent with previous work. We also compute a CMFGEN model to constrain the progenitor spectral type; the high $\dot{M}$ and low $v_{\text {wind }}$ imply that the star had an effective temperature of $\simeq 8000 \mathrm{~K}$ immediately before the SN explosion. Our models suggest that the progenitor was either an unstable luminous blue variable or a yellow hypergiant undergoing an eruptive phase, and rule out a Wolf-Rayet star. We classify the post-explosion spectra at $15.5 \mathrm{~h}$ as XWN5(h) and advocate for the use of the prefix "X" (eXplosion) to avoid confusion between post-explosion, non-stellar spectra, and those of massive stars. We show that the XWN spectrum results from the ionization of the progenitor wind after the $\mathrm{SN}$, and that the progenitor spectral type is significantly different from the early post-explosion spectral type owing to the huge differences in the ionization structure before and after the SN event. We find the following temporal evolution: LBV/YHG $\rightarrow$ XWN5(h) $\rightarrow$ SN IIb. Future early-time spectroscopy in the UV will further constrain the properties of SN precursors, such as their metallicities.
\end{abstract}

Key words. supernovae: general - stars: evolution - supernovae: individual: SN 2013cu - stars: winds, outflows

\section{Introduction}

Core-collapse supernovae ( $\mathrm{SNe})$ are the final act in the evolution of stars more massive than about $8-9 M_{\odot}$. Determining the progenitors of these explosive events and how massive stars are linked to the different SN types are topics of major significance for several fields of astrophysics.

Numerous techniques have been employed to constrain the nature of SN progenitors. For instance, direct detection of progenitors in pre-explosion images has yielded the determination of the photospheric properties of the progenitors, such as effective temperatures and luminosities (e.g., Smartt 2009). However, pre-explosion imaging provides weak direct constraints on the progenitor chemical abundance, mass loss, and wind speed. Mass loss immediately before the SN explosion has a major impact on the SN properties (see Smith 2014) and has been investigated in SN IIn using spectroscopy and photometry several days after their discoveries (e.g., Smith et al. 2007; Pastorello et al. 2007; Kiewe et al. 2012; Ofek et al. 2014; Moriya et al. 2014), and in SN IIb and Ibc using X-ray and radio observations (e.g., Chevalier \& Fransson 2006; Soderberg et al. 2012).

Recent progress in observational techniques now allow for rapid-response spectroscopic observations of SNe within a day of detection (Gal-Yam et al. 2014, hereafter G14). This allows the study of early phases when the SN shock front has not yet reached spatial scales of $10^{14} \mathrm{~cm}$. Depending on the progenitor's wind density and SN shock front velocity, these early-time $\mathrm{SN}$ observations may probe epochs early enough that the dense parts of the progenitor wind and circumstellar medium (CSM) have not yet been overrun by the SN shock front.

This was the case for the type IIb SN 2013cu (iPTF13ast) reported by G14. The spectroscopic observations obtained $15.5 \mathrm{~h}$ after first detection reveal surprising features that apparently resemble those seen in Wolf-Rayet (WR) stars of the WN6(h) subtype. Based on this spectrum, G14 suggested a WR-like progenitor with evidence of $\mathrm{H}$ in its wind, thus being consistent with the SN IIb classification obtained from later spectra. These authors also found evidence of enhanced mass loss prior to the SN explosion, with a mass-loss rate of $\dot{M} \sim 0.01 M_{\odot} \mathrm{yr}^{-1}$.

Here we present the first radiative transfer modeling of spectra of the early-time interactions of a SN with its dense progenitor wind. Our goal is to constrain the progenitor properties of SN 2013cu, such as its chemical composition, mass-loss rate, and wind velocity, from detailed modeling of spectroscopic features. As we discuss below, one of our most important findings is that the progenitor spectral type does not correspond to the spectral type detected in rapid-response, post-explosion spectra. In the case of $2013 \mathrm{cu}$, our models indicate that the progenitor was a luminous blue variable (LBV) or yellow hypergiant (YHG).

\section{Early-time modeling of SN 2013cu}

We employ the non-local thermodynamical equilibrium, spherical, line-blanketed, atmospheric/wind radiative transfer code CMFGEN (Hillier \& Miller 1998) to investigate the early 
post-explosion properties of SN 2013cu via spectroscopic modeling. At this point, we perform no hydrodynamical modeling. Our models are specified by the location of the inner boundary $\left(R_{\text {in }}\right)$, bolometric luminosity $\left(L_{\mathrm{SN}}\right)$, constant progenitor mass-loss rate $(\dot{M})$, and wind velocity $\left(v_{\text {wind }}\right)$, and $\mathrm{H}, \mathrm{He}, \mathrm{C}$, $\mathrm{N}$, and $\mathrm{O}$ abundances. Solar abundances are used for $\mathrm{P}, \mathrm{S}$, and $\mathrm{Fe}$.

We assume a steep density gradient with a scale height of $0.007 R_{\text {in }}$ to simulate the shock layer, which is joined smoothly to the non-shocked progenitor wind characterized by a density profile $\rho \propto r^{-2}$. We assume diffusion approximation at the inner boundary, which has a density $\rho_{\text {in }}$ that is adjusted to obtain a Rosseland optical depth ( $\left.\tau_{\text {ross }}\right)$ of 50 at $R_{\text {in }}$. This ensures that the photons are thermalized at all wavelengths at the inner boundary (see also Dessart \& Hillier 2005, although their models are for non-interacting SN at later epochs). These conditions mimic those of SN shock breakout that occurs in the progenitor wind or circumstellar medium (CSM; see Ofek et al. 2010; Chevalier \& Irwin 2011; Moriya \& Maeda 2014), as proposed by G14 for SN 2013cu. We also assume that no energy is generated in the progenitor wind, that time-dependent effects are negligible, and that the medium in unclumped. By fitting an observed spectrum, we are able to constrain the following properties, using the diagnostics in parenthesis:

- progenitor $\dot{M}$ (strength of the $\mathrm{H} \alpha$ line);

- progenitor $v_{\text {wind }}$ (width of the narrow component of emission lines);

- $L_{\mathrm{SN}}$ and $R_{\text {in }}$ (ionization structure and absolute flux assuming a distance $d$ to the $\mathrm{SN}$ );

- progenitor chemical abundances (H $\alpha$, He II $\lambda 5411$, C IV $\lambda \lambda 5801-5810$, N IV $\lambda \lambda 7109-7123)$.

We are aware that our post-explosion modeling has several simplifications and caveats that may affect the observables. First, we consider a stationary wind for the progenitor with constant mass-loss rate. Second, the hydrodynamics of the inner wind may be modified by the huge radiation field from the SN shock breakout as well as the radiation from the wind-SN interaction (Ofek et al. 2010; Nakar \& Sari 2010; Chevalier \& Irwin 2011; Moriya et al. 2011), with the inner wind being accelerated (Fransson et al. 2014). Radiation hydrodynamical simulations of SN interacting with a dense CSM show that acceleration of the inner wind may occur depending on the wind density and SN luminosity (Moriya et al. 2011). However, CMFGEN cannot handle non-monotonic outflows at the moment. Third, the radiation field and level populations may be affected by time dependence, since the physical conditions rapidly change within the first day after shock breakout (e.g., Dessart \& Hillier 2011; Dessart et al. 2011; Gezari et al. 2008). However, as long as the photosphere (defined as the layer where $\tau_{\text {ross }}=2 / 3$ ) is located in the progenitor wind and the SN shock front is located at high optical depths, our working model allows us to obtain realistic quantitative information about $\mathrm{SN}$ progenitor winds via spectroscopic modeling.

Figure 1a shows the observed optical spectrum of $2013 \mathrm{cu}$ at $15.5 \mathrm{~h}$ after the explosion (G14) and our best-fitting CMFGEN model. We obtain that a slow, dense wind or CSM surrounds the progenitor at the time of explosion, with $v_{\text {wind }} \lesssim 100 \mathrm{~km} \mathrm{~s}^{-1}$ and

$\dot{M} \simeq 3 \times 10^{-3}\left(\frac{v_{\text {wind }}}{100 \mathrm{~km} \mathrm{~s}^{-1}}\right) M_{\odot} \mathrm{yr}^{-1}$.

We note that lower values of $v_{\text {wind }}$ are allowed since the narrow component of the spectral lines is unresolved by the observations. Our values of $\dot{M}$ and $v_{\text {wind }}$ are lower than the analytical

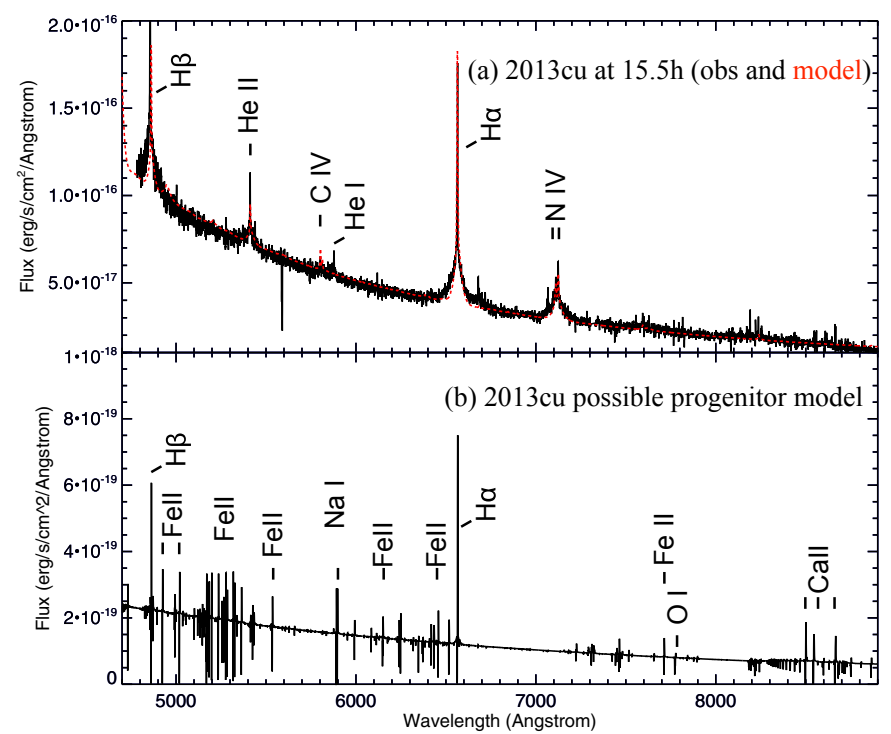

Fig. 1. a) Model of the spectrum of SN 2013cu at $15.5 \mathrm{~h}$ after the explosion (red-dashed line) and the respective observations from G14 (black). The strongest features are labeled. b) Example of a possible LBV preexplosion spectrum of the progenitor of SN 2013cu. We note the huge difference in pre- and post-explosion morphology due to very different ionization conditions before and after the SN.

estimates of $\mathrm{G} 14\left(1 \times 10^{-2} M_{\odot} \mathrm{yr}^{-1}\right.$ and $500 \mathrm{~km} \mathrm{~s}^{-1}$, respectively). This difference arises from our detailed line-profile modeling, which includes an accurate treatment of electron scattering. We refer the reader to Dessart et al. (2009) for a discussion on how CMFGEN treats electron scattering. Still, our determination of $\dot{M} / v_{\infty}$ is roughly consistent with this previous study. Our best-fitting model has $L_{\mathrm{SN}}=1 \times 10^{10} L_{\odot}$ and $R_{\text {in }}=1.5 \times 10^{14} \mathrm{~cm}$, which is consistent with the dense portions of the wind not being overrun by the SN shock front.

The early-time spectral morphology of SN 2013cu is accurately reproduced by our model (Fig. 1a). We can reproduce reasonably well the strength and shape of most features, with a narrow component and broad emission wings that are caused by electron scattering and not by the wind velocity field. The blue wings of the Balmer lines are underestimated by our models, which may be a sign that the inner wind is accelerated (Fransson et al. 2014). The strength of the He I lines is also underestimated by our models, although a larger value of $R_{\text {in }}$ would reproduce the He I lines more accurately.

Our modeling allows us to directly determine the chemical composition of a $\mathrm{SN}$ progenitor wind. We find a significant amount of $\mathrm{H}$ and $\mathrm{He}$, with abundances (by mass) of $X \approx$ $0.46 \pm 0.2$ and $Y \approx 0.52 \pm 0.2$, respectively. The errors are largely dominated by the unsatisfactory fit of the He I lines, and future model improvements could possibly reduce these uncertainties significantly. We find that the wind is enhanced in $\mathrm{N}$ and depleted in $\mathrm{C}$ relative to solar values (mass fractions of $8.0 \times 10^{-3}$ and $1.0 \times 10^{-5}$, respectively). Assuming a solar CNO content yields a depleted $\mathrm{O}$ mass fraction of $1.6 \times 10^{-4}$. We estimate the error in the CNO abundances to be $50 \%$. The errors in the abundances are determined by running models with different abundances, comparing the synthetic spectra and observations by eye, and estimating the range of values that provide a satisfactory fit. Taking the errors into account, the abundances of $2013 \mathrm{cu}$ are consistent with having a progenitor that lost a sizable fraction of the $\mathrm{H}$ envelope and presents fully $\mathrm{CNO}$-processed material at the surface, confirming the suggestions from $\mathrm{G} 14$. 


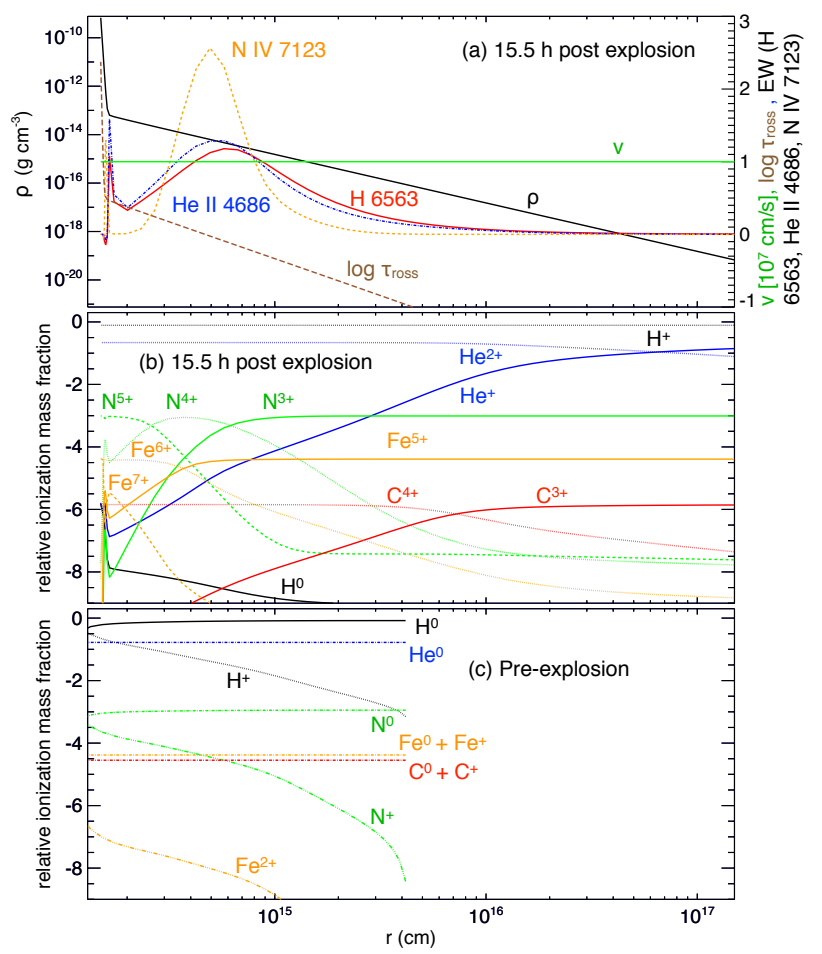

Fig. 2. a) Density, velocity, Rosseland optical depth, and formation regions of $\mathrm{H} \alpha$, He II $\lambda 5411$, and N IV $\lambda 7123$ (the area under the curve is proportional to the normalized equivalent width) of SN 2013cu based on our CMFGEN model at $15.5 \mathrm{~h}$ after the explosion. b) Predicted ionization structure at $15.5 \mathrm{~h}$ after the explosion. c) Predicted ionization structure of a possible LBV progenitor before the SN explosion.

Figure 2a shows the physical conditions of SN $2013 \mathrm{cu}$ at $15.5 \mathrm{~h}$ after explosion. We note that the photosphere is located in the progenitor wind. We find that the strongest lines in the $15.5 \mathrm{~h}$ optical spectrum, such as $\mathrm{H} \alpha$, He II $\lambda 5411$, and N IV $\lambda 7123$, are formed over an extended distance of 2-20 $\times$ $10^{14} \mathrm{~cm}$ (containing $2.2 \times 10^{-2} M_{\odot}$ ), with the bulk of the emission coming from $4-7 \times 10^{14} \mathrm{~cm}$ (containing $4.5 \times 10^{-3} M_{\odot}$ ). We also obtain that the outflow is significantly ionized, with $\mathrm{H}$ and He being fully ionized up to large distances $\left(\gtrsim 10^{16} \mathrm{~cm}\right)$. The ionization structure at $15.5 \mathrm{~h}$ post-explosion is shown in Fig. $2 \mathrm{~b}$.

Although an apparent WN(h)-like spectrum is seen at $15.5 \mathrm{~h}$ after the explosion, we argue here that the spectral type of the progenitor was not the same as that seen during the postexplosion because of the very different ionization conditions at the pre- and post-explosion epochs (Fig. 2b,c). Since the number of ionizing photons at the inner boundary at $15.5 \mathrm{~h}$ is many orders of magnitude larger than the typical number of ionizing photons of SN progenitors, the progenitor wind is quickly ionized. Our model shows that the ionization structure after the $\mathrm{SN}$ explosion responds to the new $\mathrm{SN}$ effective temperature and huge increase in luminosity (Fig. 2b).

Because of the non-stellar origin of the apparent $\mathrm{WN}(\mathrm{h})$ post-SN explosion spectrum, we advocate here for the use of the prefix "X" (short for eXplosion) before the spectral type of such events like SN and SN impostors where a shock breakout and/or interacting CSM illuminate and ionize the precursor wind. This will avoid future confusion between spectral types seen after the explosion with those of their progenitors. Thus, in the case of the SN 2013cu spectrum at $15.5 \mathrm{~h}$, the spectral type would be XWN5(h), following the WN classification scheme of Smith et al. (1996) and noting that the C IV $\lambda 5808 / \mathrm{He}$ II $\lambda 5411$ ratio favors the earlier classification.

\section{A luminous blue variable or yellow hypergiant precursor directly before SN 2013cu}

To investigate the spectral morphology of the progenitor, we compute its predicted spectrum using as input the values obtained from the post-explosion spectral modeling $(\dot{M} \simeq 3 \times$ $10^{-3} M_{\odot} \mathrm{yr}^{-1}, v_{\text {wind }} \simeq 100 \mathrm{~km} \mathrm{~s}^{-1}$, and the aforementioned chemical abundances). We use CMFGEN in its standard stellar mode as described in, e.g., Groh et al. (2009), where a hydrostatic solution is computed for the subsonic region and merged with the wind solution at 0.75 of the sonic speed. The progenitor wind is assumed to accelerate following a beta-type law with $\beta=1$. Since we have no constraints on the luminosity $\left(L_{\star}\right)$ and hydrostatic radius $\left(R_{\star}\right)$ of the progenitor, we assume $R_{\star}=100 R_{\odot}$ and $L_{\star}=5 \times 10^{5} L_{\odot}$ to compute its spectrum. Owing to the large $\dot{M}$ and low $v_{\text {wind }}$ inferred from the post-explosion spectrum, our pre-explosion models predict that the progenitor has a pseudo-photosphere with $T_{\text {eff }} \simeq 8000 \mathrm{~K}$. The qualitative preexplosion spectral morphology and $T_{\text {eff }}$ are only weakly dependent on $L_{\star}$ and $R_{\star}$ for sensible changes in these values, i.e., even a compact progenitor would show an LBV morphology immediately before the $\mathrm{SN}$ explosion. A similar situation where the hydrostatic layers are deeply buried in an optically thick wind is seen in Eta Carinae (Hillier et al. 2001, 2006; Groh et al. 2012).

Figure $1 \mathrm{~b}$ shows a prediction of the possible pre-explosion optical spectrum. The pre- and post-explosion spectra differ significantly because of the different $T_{\text {eff }}$, luminosity, and ionization structures in the two situations (Figs. 2b,c). This example of pre-explosion spectrum resembles those seen in luminous blue variables (LBVs), which are a class of unstable massive stars that are found both as a transitional stage from an O-type to a WR star (Humphreys \& Davidson 1994; Groh et al. 2014) and as SN progenitors (Kotak \& Vink 2006; Smith et al. 2007, 2011; Kochanek et al. 2011; Pastorello et al. 2007; Trundle et al. 2008; Gal-Yam \& Leonard 2009; Groh \& Vink 2011; Mauerhan et al. 2013; Ofek et al. 2013; Groh et al. 2013a,b).

Given our lack of constraints on $R_{\star}$, another possibility is that the progenitor was a cool yellow hypergiant (YHG) with $T_{\text {eff }} \simeq 5000-7000 \mathrm{~K}$ (see Groh et al. 2013b). In this case, the progenitor would be extended $\left(R_{\star}>10^{12} \mathrm{~cm}\right)$. The progenitor would be losing mass in an outburst similar to that of the Galactic YHG $\rho$ Cas in the year 2000-2001 (Lobel et al. 2003), which further strengthens the link between SN 2013cu and YHGs. Of crucial importance here is that the progenitor of this SN IIb would be a YHG, and not a yellow supergiant (YSG) as often quoted in the literature (e.g., Maund et al. 2011; Van Dyk et al. 2013) because YHGs have extensive mass loss and outbursts (de Jager 1998), which is exactly what we infer for the progenitor of SN 2013cu (see discussion in Groh et al. 2013b).

While the hydrostatic radius of SN IIb progenitors have been constrained by hydrodynamical modeling of the SN lightcurve (e.g., Bersten et al. 2012), this may be challenging for SN 2013cu because of circumstellar interaction. Even then, it would be hard to differentiate between LBVs and YHGs, since they both share similar values of radii (up to several $10^{13} \mathrm{~cm}$ ). To differentiate between LBVs and cool YHGs, constraints on the luminosity and/or effective temperature of the progenitor would be needed, but this is only directly accessible by detecting the progenitor in pre-explosion images. For distant events as in the case of $2013 \mathrm{cu}(d=108 \mathrm{Mpc})$, however, this would be challenging with the current instrumentation.

Therefore, we argue that the high value of $\dot{M}$, relatively low $v_{\text {wind }}$, and chemical abundance pattern are consistent with the progenitor being either an LBV or YHG (and not a WN star) 
A\&A 572, L11 (2014)

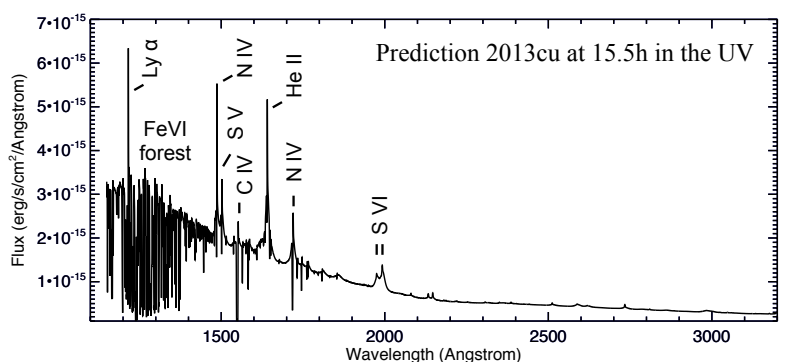

Fig. 3. Prediction of the early UV spectrum of SN 2013cu based on our CMFGEN model at $15.5 \mathrm{~h}$ after the explosion. A reddening of $A_{V}=$ $0.1 \mathrm{mag}$ is assumed.

immediately before the SN explosion. It may be possible that the star had a totally different spectral morphology before the LBV or YHG eruption phase. Based on the chemical abundances, at this pre-LBV/YHG eruptive phase, the progenitor could have been a WN, LBV, blue supergiant, YHG, or yellow supergiant, depending on its $R_{\star}$ and $\dot{M}$. Time-dependent modeling of the SN 2013cu spectral evolution is warranted to investigate the rapid evolution before core collapse. In conclusion, we obtain the following temporal evolution for SN 2013cu and its progenitor:

$$
\left.\begin{array}{lcc}
\mathrm{LBV} / \mathrm{YHG} \\
(\text { pre }-\mathrm{SN})
\end{array} \quad \rightarrow \quad \text { XWN5(h) } \quad \rightarrow \quad \begin{array}{c}
\text { SNIIb } \\
(15.5 \text { hpost }-\mathrm{SN})
\end{array} \quad \text { (69dpost }-\mathrm{SN}\right) .
$$

\section{Prospects with UV spectroscopy and outlook}

The optical spectra of SN 2013cu, although it has a large wavelength coverage, is relatively poor in bright emission lines. Having access to more spectral lines would significantly aid the determination of the progenitor properties, and this would best be achieved by observing in the UV.

Ultraviolet spectroscopy offers several other advantages, such as allowing one to observe as close as possible to the peak of the spectral energy distribution of SNe at early epochs. Our predicted spectrum indicates a flux an order of magnitude higher in the UV at $15.5 \mathrm{~h}$ (Fig. 3). Observing in the UV allows one to probe resonance lines, such as C IV $\lambda 1548-1551$, and other strong features such as He II $\lambda 1640$, and N IV $\lambda 1718$. Given their high optical depths, observing UV resonance lines should provide detectable spectral features even at much lower wind densities than those of SN 2013cu. In addition, these lines allow a more precise determination of the velocity structure of the progenitor wind and $\mathrm{SN}$-wind interacting region, better constraining the models. Our models predict that many Fe VI and Fe VII lines should be detectable at $\lambda \lambda 1200-1450$. These have the potential to be used as direct probes of the progenitor and SN metallicities. We note that, in this particular case, the predicted spectrum is almost featureless in the range 2000-3000 $\AA$, suggesting that observations should be focused below $2000 \AA$.

Early spectroscopy of $\mathrm{SNe}$ opens up a new observational window that allows the inference of progenitor properties shortly before explosion. We have shown that when these recent observations are combined with non-LTE radiative transfer modeling, quantitative information can be obtained about the progenitor, such as its chemical composition, wind velocity, and mass-loss rate. These quantities allow us to better infer the nature of the progenitor, in particular because our implementation allows the computation of a realistic progenitor spectrum, as illustrated in Fig. 1b.

In a general case, the apparent spectral morphology at early times will change according to the progenitor wind density and composition, time after SN shock breakout, and SN luminosity. We predict that this will produce a greater variety of spectral morphologies as the number of events with early-time spectra increases.

For the new events, early-time SN spectroscopy has the potential to provide even more constraints on the progenitor properties if combined with hydrodynamical modeling of the SN lightcurve and/or fortuitous pre-explosion imaging of the progenitor. Having progenitors detected in pre-explosion imaging and spectroscopically observed within a day after the SN explosion will allow us to put definitive constraints on their luminosities, effective temperatures, chemical abundances, wind velocities and mass-loss rates. This will be an important step towards a more complete understanding of the diversity of SN types and the evolutionary channels that produce their progenitors.

Acknowledgements. J.H.G. is supported by an Ambizione Fellowship of the Swiss National Science Foundation. J.H.G. thanks A. Gal-Yam, O. Yaron, and E. Ofek for making the observed spectrum of SN 2013cu available, valuable discussions, and the warm hospitality at the Weizmann Institute of Science (Israel). Discussions and comments from J. Hiller, T. Moriya, N. Smith, M. Bersten, and L. Dessart are also acknowledged, as are the comments provided by the anonymous referee.

\section{References}

Bersten, M. C., Benvenuto, O. G., Nomoto, K., et al. 2012, ApJ, 757, 31 Chevalier, R. A., \& Fransson, C. 2006, ApJ, 651, 381

Chevalier, R. A., \& Irwin, C. M. 2011, ApJ, 729, L6 de Jager, C. 1998, A\&ARv, 8, 145

Dessart, L., \& Hillier, D. J. 2005, A\&A, 437, 667

Dessart, L., \& Hillier, D. J. 2011, MNRAS, 410, 1739

Dessart, L., Hillier, D. J., Gezari, S., Basa, S., \& Matheson, T. 2009, MNRAS 394,21

Dessart, L., Hillier, D. J., Livne, E., et al. 2011, MNRAS, 414, 2985

Fransson, C., Ergon, M., Challis, P. J., et al. 2014, ApJ, in press [arXiv: 1312.6617]

Gal-Yam, A., \& Leonard, D. C. 2009, Nature, 458, 865

Gal-Yam, A., Arcavi, I., Ofek, E. O., et al. 2014, Nature, 509, 471

Gezari, S., Dessart, L., Basa, S., et al. 2008, ApJ, 683, L131

Groh, J. H., \& Vink, J. S. 2011, A\&A, 531, L10

Groh, J. H., Hillier, D. J., Damineli, A., et al. 2009, ApJ, 698, 1698

Groh, J. H., Hillier, D. J., Madura, T. I., \& Weigelt, G. 2012, MNRAS, 423, 1623

Groh, J. H., Meynet, G., \& Ekström, S. 2013a, A\&A, 550, L7

Groh, J. H., Meynet, G., Georgy, C., \& Ekström, S. 2013b, A\&A, 558, A131

Groh, J. H., Meynet, G., Ekström, S., \& Georgy, C. 2014, A\&A, 564, A30

Hillier, D. J., \& Miller, D. L. 1998, ApJ, 496, 407

Hillier, D. J., Davidson, K., Ishibashi, K., \& Gull, T. 2001, ApJ, 553, 837

Hillier, D. J., Gull, T., Nielsen, K., et al. 2006, ApJ, 642, 1098

Humphreys, R. M., \& Davidson, K. 1994, PASP, 106, 1025

Kiewe, M., Gal-Yam, A., Arcavi, I., et al. 2012, ApJ, 744, 10

Kochanek, C. S., Szczygiel, D. M., \& Stanek, K. Z. 2011, ApJ, 737, 76

Kotak, R., \& Vink, J. S. 2006, A\&A, 460, L5

Lobel, A., Dupree, A. K., Stefanik, R. P., et al. 2003, ApJ, 583, 923

Mauerhan, J. C., Smith, N., Filippenko, A., et al. 2013, MNRAS, 430, 1801

Maund, J. R., Fraser, M., Ergon, M., et al. 2011, ApJ, 739, L37

Moriya, T. J., \& Maeda, K. 2014, ApJ, 790, L16

Moriya, T., Tominaga, N., Blinnikov, S. I., Baklanov, P. V., \& Sorokina, E. I 2011, MNRAS, 415, 199

Moriya, T. J., Maeda, K., Taddia, F., et al. 2014, MNRAS, 439, 2917

Nakar, E., \& Sari, R. 2010, ApJ, 725, 904

Ofek, E. O., Rabinak, I., Neill, J. D., et al. 2010, ApJ, 724, 1396

Ofek, E. O., Sullivan, M., Cenko, S. B., et al. 2013, Nature, 494, 65

Ofek, E. O., Sullivan, M., Shaviv, N. J., et al. 2014, ApJ, 789, 104

Pastorello, A., Smartt, S. J., Mattila, S., et al. 2007, Nature, 447, 829

Smartt, S. J. 2009, ARA\&A, 47, 63

Smith, N. 2014, ARA\&A, 52,487

Smith, L. F., Shara, M. M., \& Moffat, A. F. J. 1996, MNRAS, 281, 163

Smith, N., Li, W., Foley, R. J., et al. 2007, ApJ, 666, 1116

Smith, N., Li, W., Miller, A. A., et al. 2011, ApJ, 732, 63

Soderberg, A. M., Margutti, R., Zauderer, B. A., et al. 2012, ApJ, 752, 78

Trundle, C., Kotak, R., Vink, J. S., \& Meikle, W. P. S. 2008, A\&A, 483, L47

Van Dyk, S. D., Zheng, W., Clubb, K. I., et al. 2013, ApJ, 772, L32 